\title{
Crystal structure of bis(3,5-dimethylanilinium) dihydrogenodiphosphate, $\left(\mathrm{C}_{8} \mathrm{H}_{12} \mathrm{~N}\right)_{2} \mathrm{H}_{2} \mathrm{P}_{2} \mathrm{O}_{7}$
}

\section{S. Akriche*}

Faculté des Sciences de Bizerte, Laboratoire de Chimie des Matériaux, 7021 Zarzouna Bizerte, Tunesia

Received January 7, 2003, accepted and available on-line February 1, 2003; CCDC-No. 1267/1006

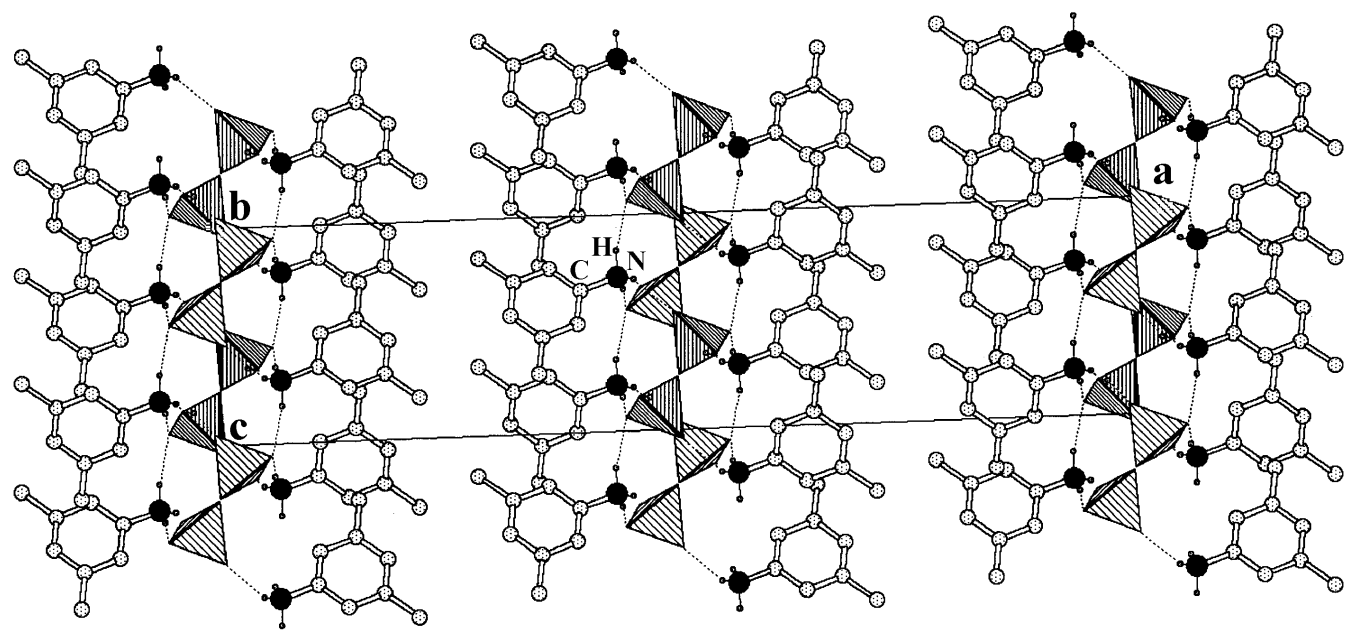

Abstract

$\mathrm{C}_{16} \mathrm{H}_{26} \mathrm{~N}_{2} \mathrm{O}_{7} \mathrm{P}_{2}$, monoclinic, $C 12 / c 1$ (No. 15), $a=32.53(1) \AA$, $b=8.415(2) \AA, c=7.792(4) \AA, \beta=92.18(3)^{\circ}, V=2131.4 \AA^{3}$, $Z=4, R_{\mathrm{gt}}(F)=0.071, w R_{\mathrm{ref}}(F)=0.077, T=296 \mathrm{~K}$.

\section{Source of material}

The diphosphoric acid was prepared by passing a concentrated solution of sodium diphosphate $\mathrm{Na}_{4} \mathrm{P}_{2} \mathrm{O}_{7}$ through an ion exchange resin (Amberlite IR 120) in its $\mathrm{H}$-state. The diphosphoric acid was then neutralized with 3,5-dimethylaniline at low temperature by mixing them in stochiometric proportions. The resulting solution was slowly evaporated at room temperature until the formation of crystals of $\left(\mathrm{C}_{8} \mathrm{H}_{12} \mathrm{~N}\right)_{2} \mathrm{H}_{2} \mathrm{P}_{2} \mathrm{O}_{7}$.

\section{Experimental details}

The slightly high value of $R$ is due to the bad quality of the crystal.

\section{Discussion}

The crystal structure of the title compound can be described as a succession of thick layers perpendicular to the $a$ axis at $x=0$ and $x$ $=1 / 2$. These layers are formed by phosphoric $\mathrm{H}_{2} \mathrm{P}_{2} \mathrm{O}_{7}{ }^{2-}$ and organic 3,5- $\left(\mathrm{CH}_{3}\right)_{2} \mathrm{C}_{6} \mathrm{H}_{4} \mathrm{NH}_{3}$ entities. Each $\mathrm{H}_{2} \mathrm{P}_{2} \mathrm{O}_{7}{ }^{2-}$ anion is connected to two adjacent neighbor diphosphate groups by strong hydrogen bonds building corrugated ribbons parallel to the $c$ direction. These ribbons are interconnected by the H-bonds created by ammonium groups of organic cations. The $\mathrm{H}_{2} \mathrm{P}_{2} \mathrm{O}_{7}$ entity has a two-fold internal symmetry. The bridging oxygen atom is located on the two-fold axis. The empirical formula of the title compound is similar to that of another published diphosphate [1]. However, the two compounds are

\footnotetext{
* Correspondence author (e-mail: Mohamed.Rzaigui@fsb.rnu.tn)
}

completely different. Their organic molecules (3,5-dimethylanilinium for one and 2-phenylethylammonium for the other) and their crystallographic data are not comparable.

Table 1. Data collection and handling.
Crystal:

Wavelength:

$\mu$ :

Diffractometer, scan mode:

$2 \theta_{\max }$ :

$N(h k l)_{\text {measured, }} N(h k l)_{\text {unique: }}$

Criterion for $I_{\mathrm{obs}}, N(h k l)_{\mathrm{gt}}$ :

$N(\text { param })_{\text {refined: }}$

Programs: colorless prism, size $0.15 \times 0.20 \times 0.35 \mathrm{~mm}$ Mo $K_{\alpha}$ radiation $(0.7107 \AA)$

$2.41 \mathrm{~cm}^{-1}$

$\mathrm{MACH} 3, \omega / 2 \theta$

$59.92^{\circ}$

3739,3280

$I_{\mathrm{obs}}>2 \sigma\left(I_{\mathrm{obs}}\right), 2193$

123

teXsan [2], SIR92 [3]
Table 2. Atomic coordinates and displacement parameters (in $\AA^{2}$ ).

\begin{tabular}{llllll}
\hline Atom & Site & $x$ & $y$ & $z$ & $U_{\text {iso }}$ \\
\hline $\mathrm{H}(1)$ & $8 f$ & 0.4702 & 1.0248 & 0.8473 & 0.047 \\
$\mathrm{H}(2)$ & $8 f$ & 0.4516 & 0.6990 & 0.3002 & 0.058 \\
$\mathrm{H}(3)$ & $8 f$ & 0.4325 & 0.6064 & 0.1690 & 0.044 \\
$\mathrm{H}(4)$ & $8 f$ & 0.4397 & 0.5251 & 0.3464 & 0.057 \\
$\mathrm{H}(5)$ & $8 f$ & 0.4106 & 0.7648 & 0.5749 & 0.034 \\
$\mathrm{H}(6)$ & $8 f$ & 0.3688 & 0.9181 & 0.7657 & 0.133 \\
$\mathrm{H}(7)$ & $8 f$ & 0.3269 & 0.9571 & 0.7160 & 0.132 \\
$\mathrm{H}(8)$ & $8 f$ & 0.3379 & 0.8074 & 0.7991 & 0.144 \\
$\mathrm{H}(9)$ & $8 f$ & 0.2917 & 0.7775 & 0.4962 & 0.070 \\
$\mathrm{H}(10)$ & $8 f$ & 0.2641 & 0.7270 & 0.1803 & 0.164 \\
$\mathrm{H}(11)$ & $8 f$ & 0.2706 & 0.5514 & 0.2383 & 0.166 \\
$\mathrm{H}(12)$ & $8 f$ & 0.2884 & 0.6006 & 0.0751 & 0.144 \\
$\mathrm{H}(13)$ & $8 f$ & 0.3614 & 0.5865 & 0.1309 & 0.050 \\
\hline
\end{tabular}


Table 3. Atomic coordinates and displacement parameters (in $\AA^{2}$ ).

\begin{tabular}{|c|c|c|c|c|c|c|c|c|c|c|}
\hline Atom & Site & $x$ & $y$ & $z$ & $U_{11}$ & $U_{22}$ & $U_{33}$ & $U_{12}$ & $U_{13}$ & $U_{23}$ \\
\hline $\mathrm{P}(1)$ & $8 f$ & $0.47650(3)$ & $0.7779(1)$ & $0.9070(2)$ & $0.0357(6)$ & $0.0223(5)$ & $0.0264(5)$ & $-0.0009(6)$ & $0.0008(4)$ & $-0.0006(6)$ \\
\hline $\mathrm{O}(1)$ & $8 f$ & $0.45777(9)$ & $0.9354(4)$ & $0.8363(4)$ & $0.041(2)$ & $0.019(2)$ & $0.051(2)$ & $0.003(1)$ & $-0.009(2)$ & $-0.005(2)$ \\
\hline $\mathrm{O}(2)$ & $8 f$ & $0.50699(9)$ & $0.8069(4)$ & $1.0501(4)$ & $0.049(2)$ & $0.025(2)$ & $0.032(2)$ & $0.005(1)$ & $-0.005(1)$ & $-0.003(1)$ \\
\hline $\mathrm{O}(3)$ & $4 e$ & $1 / 2$ & $0.7016(5)$ & $3 / 4$ & $0.060(3)$ & $0.025(3)$ & $0.038(2)$ & 0 & $0.018(2)$ & 0 \\
\hline $\mathrm{O}(4)$ & $8 f$ & $0.44302(9)$ & $0.6636(4)$ & $0.9428(4)$ & $0.041(2)$ & $0.038(2)$ & $0.037(2)$ & $-0.010(1)$ & $0.007(1)$ & $-0.001(2)$ \\
\hline $\mathrm{N}(1)$ & $8 f$ & $0.4333(1)$ & $0.6220(5)$ & $0.2893(5)$ & $0.030(2)$ & $0.030(2)$ & $0.036(2)$ & $-0.002(2)$ & $0.002(2)$ & $-0.001(2)$ \\
\hline$C(2)$ & $8 f$ & $0.3899(1)$ & $0.7474(6)$ & $0.4987(6)$ & $0.043(3)$ & $0.050(4)$ & $0.040(3)$ & $-0.002(2)$ & $0.003(2)$ & $-0.007(3)$ \\
\hline $\mathrm{C}(3)$ & $8 f$ & $0.3513(2)$ & $0.7891(8)$ & $0.5581(7)$ & $0.050(3)$ & $0.069(4)$ & $0.058(3)$ & $0.000(3)$ & $0.014(3)$ & $-0.014(4)$ \\
\hline $\mathrm{C}(4)$ & $8 f$ & $0.3171(1)$ & $0.7513(8)$ & $0.4541(8)$ & $0.038(3)$ & $0.071(5)$ & $0.075(4)$ & $0.011(3)$ & $0.013(3)$ & $-0.014(4)$ \\
\hline$C(5)$ & $8 f$ & $0.3199(2)$ & $0.6753(8)$ & $0.3009(8)$ & $0.033(3)$ & $0.094(5)$ & $0.070(4)$ & $-0.002(3)$ & $-0.007(3)$ & $-0.010(4)$ \\
\hline$C(6)$ & $8 f$ & $0.3586(2)$ & $0.6332(7)$ & $0.2426(7)$ & $0.036(3)$ & $0.073(4)$ & $0.049(3)$ & $0.010(3)$ & $-0.004(2)$ & $-0.014(3)$ \\
\hline$C(7)$ & $8 f$ & $0.3479(2)$ & $0.875(1)$ & $0.725(1)$ & $0.063(5)$ & $0.162(9)$ & $0.106(6)$ & $0.008(5)$ & $0.014(4)$ & $-0.072(6)$ \\
\hline $\mathrm{C}(8)$ & $8 f$ & $0.2822(2)$ & $0.633(1)$ & $0.184(1)$ & $0.049(4)$ & $0.20(1)$ & $0.126(7)$ & $0.017(5)$ & $-0.025(4)$ & $-0.069(7)$ \\
\hline
\end{tabular}

\section{References}

1. Hlel, F.; Smiri, L.: Synthesis and crystal structure of bis(2-phenylethylammonium) dihydrogendiphosphate $\left[\mathrm{C}_{6} \mathrm{H}_{4}\left(\mathrm{CH}_{2}\right)_{2} \mathrm{NH}_{3}\right]_{2} \mathrm{H}_{2} \mathrm{P}_{2} \mathrm{O}_{7}$. Solid State Sciences 1 (1999) 321-329.

2. teXsan for Windows. Single Crystal Structure Analysis Software. Version 1.03. Molecular Structure Corporation (MSC). 3200 Research Forest Drive, The Woodlands, TX 77381, USA 1997.
3. Altomare, A.; Cascarano, M.; Giacovazzo, C.; Guagliardi, A.; Burla, M, C.; Polidori, G.; Carnalli, M.: SIR92 a program for automatic solution of crystalstructure by direct methods. J. Appl. Crystallogr. 27 (1994) 435. 